\title{
Die französische Kommunikationswissenschaft, semiotische Perspektiven und die Sphären der Postmoderne
}

\author{
Stefanie Averbeck
}

„Sind denn alle französischen Kommunikationswissenschaftler Postmodernisten?“, fragen Siegfried J. Schmidt und Joachim Westerbarkey in ihrer Replik auf Ulrich Saxers „Mythos Postmoderne“.1 Die Frage ist berechtigt, aber sie führt noch weiter: Denn Baudrillard, Virilio, Lyotard und die anderen von Saxer genannten befassen sich zwar (auch) mit Kommunikation, aber sie sind keine Kommunikationswissenschaftler im Sinne derer, die das Fach an französischen Universitäten lehren.

Wer also sind die französischen Kommunikationswissenschafter und welche Positionen vertreten sie? Hierzulande kennt man die Namen französischer Kommunikationswissenschaftler zumeist nicht: Francis Balle, Universität Paris (Panthéon Assas), Armand Mattelart, Universität Rennes, Daniel Bougnoux, Universität Grenoble, Jean Jacques Boutaud, Professor für Kommunikationswissenschaft in Bourgogne, um nur einige wenige zu nennen.2 Balles Buch „Médias et Sociétés“, laut seines Pariser Kollegen Jean Cazeneuve das zentrale systematische Werk der französischen Kommunikationswissenschaft, ist in Frankreich inzwischen in neunter Auflage erschienen, ${ }^{3}$ immerhin erhält man es in englischer Übersetzung, aber das ist schon die Ausnahme. Die Wahrnehmung der französischen Kommunikationswissenschaft ist, wie umgekehrt die der deutschen in Frankreich, insbesondere ein Sprachproblem. Saxers Leseaufforderung an die deutschen Kommunikationswissenschaftler - „da ja Übersetzungen vorliegen “4 - ist zu lapidar und betrifft fast ausschließlich Soziologen und Philosophen mit hohem weltweiten Renommee wie Jacques Derrida, Paul Virilio oder Jean Baudrillard, aber eben nicht Kommunikationswissenschaftler.

1 Ulrich Saxer: Mythos Postmoderne: Kommunikationswissenschaftliche Bedenken. In: Medien \& Kommunikationswissenschaft, 48. Jg. 2000, Nr. 1, S. 85 - 92; Siegfried J. Schmidt/Joachim Westerbarkey: Mehr Querschläger als Blattschuss: Eine Replik auf Ulrich Saxers Philippika wider postmoderne Kommunikationstheoreme. In: Medien \& Kommunikationswissenschaft, 48. Jg. 2000, Nr. 2, S. $247-251$.

2 Vgl. die interdisziplinär orientierte Aufstellung über Wissenschaftler, die sich mit Kommunikationsforschung befassen, in: Les chercheurs en information et en communication. Annuaire 1997 - 1998. Edité par la Société Française de l'Information et de la Communication (SFSIC). Diese Aufstellung erschien seit 1992 sechsmal; 1992 führte sie 221 Forscher auf, 1998 schon 319. Es handelt sich dabei indes nicht um das Mitgliederverzeichnis der Französischen Gesellschaft für Kommunikationswissenschaft, sondern um einen von ihr erbrachten Service, der der disziplinübergreifenden Kooperation dienen soll.

3 Françis Balle: Médias et Sociétés. De Gutenberg à Internet. Presse, Audiovisuel, Télécommunications, Multimédia, Télématique. 9ième édition. Paris: Montchrestien 1999. Zu einer früheren Auflage dieses Buches vgl. Jean Cazeneuve: La France. In: Les cahiers de la communication, Vol. 2 1982, No. 4 - 5 (Numéro: Communications et médias. Les orientations de la recherche en Europe), S. 369-385.

4 Vgl. Saxer (wie Anm. 1), S. 90. 


\section{Kommunikationswissenschaft in Frankreich: Einige Anmerkungen}

Vorausgeschickt sei, dass dieser Beitrag keinen Anspruch auf eine vollständige Darstellung der französischen Kommunikationswissenschaft erhebt, sondern vor allem auf deren semiotische Richtung fokussiert, die in Deutschland weitgehend unbekannt sein dürfte. Auch versteht er sich weniger als Replik auf Ulrich Saxers „Mythos Postmoderne“ oder Schmidt/Westerbarkeys Entgegnung darauf, sondern als „Anschlusskommunikation" an beide. Denn beide Aufsätze verweisen bei aller Verschiedenheit auf ein Forschungsdesiderat „Französische Kommunikationswissenschaft“.

Das Feld der Kommunikationswissenschaft ist auch in Frankreich „transdisziplinär“5 ausgeformt. Es reicht von der Politikwissenschaft bis über die Soziologie und vor allem in die Semiotik. Ohne den stark semiotischen Zugriff der französischen Kommunikationswissenschaft nachzuvollziehen, der seinen empirischen Gegenstand in der Sprache und in der Sprachpraxis sowie den kulturell konstituierenden Mechanismen von Sinngebung findet, kann man dieses Fach in unserem Nachbarland kaum verstehen. Jean Jacques Boutaud folgend hat die Semiotik zwei empirische Bezugspunkte: Die Materialität der Zeichen und ihre innere Struktur (das Forschungsfeld der klassischen Semiologie) sowie darüber hinausgehend und primär, den kulturell definierten symbolischen Umgang der Menschen mit ihren Zeichen. ${ }^{6}$ Damit sind die Grundlagen kommunikativen Verhaltens angesprochen.

Die Semiotik als Bedeutungslehre der Zeichen im weitesten Sinne wird in der deutschsprachigen Kommunikationswissenschaft kaum beachtet, Ausnahmen sind etwa die älteren Arbeiten von Günter Bentele sowie Roland Burkarts einführende Übersichten zur allgemeinen Semiotik, insbesondere ihrer pragmatischen Richtungen.7

In der französischen Kommunikationswissenschaft gibt es keine namentlich kenntlich gemachte „konstruktivistische“ oder „radikal-konstruktivistische“ Richtung, wohl aber die bereits benannte semiotische, und beide haben möglicherweise nicht zufällig die gleiche empirische Bezugsbasis ihres Nachdenkens: Sprache und Kultur. Mit dem Verweis auf Sprache und Kultur etwa bieten Schmidt/Westerbarkey dem saxerschen Vorwurf „subjektivistischer Einseitigkeit“ des Konstruktivismus Einhalt. ${ }^{8}$ Wohl nicht zufällig ist auch, dass die semiotische Richtung in Frankreich offen ist für die Ansätze von Norbert Wiener, Gregory Bateson und Paul Watzlawick, die alle auf ihre Weise Potential für konstruktivistische Perspektiven entfalten.

5 Ein Terminus, den Ulrich Saxer für die deutschsprachige Kommunikationswissenschaft eingeführt hat; vgl. Ulrich Saxer: Basistheorien und Theoriebasis in der Kommunikationswissenschaft. Theorienchaos und Chaostheorie. München: Ölschläger 1993 (= Schriftenreihe der Deutschen Gesellschaft für Publizistik- und Kommunikationswissenschaft, Bd. 19), S. 175 $187,175$.

6 Vgl. Jean Jacques Boutaud: Sémiotique et communication. Du signe au sens. Paris: L'Harmattan 1998, S. 92. Boutaud legt die erkenntnistheoretische Relevanz der Semiotik für eine Wissenschaft von der Kommunikation detailliert dar.

7 Vgl. Günter Bentele: Zeichen und Entwicklung. Vorüberlegungen zu einer genetischen Semiotik. Tübingen: Gunter Narr 1984; Günter Bentele/Ernest W. B. Lüttich (Hrsg.): Zeichengebrauch in Massenmedien. Zum Verhältnis von sprachlicher und nichtsprachlicher Information in Hörfunk, Film und Fernsehen. Tübingen: Niemeyer 1985; Roland Burkart: Kommunikationswissenschaft. Grundlagen und Problemfelder. Umrisse einer interdisziplinären Sozialwissenschaft, 3. aktualisierte Aufl. Wien: Böhlau 1998, S. 73ff. sowie neuerdings Frank Hartmann: Medienphilosophie. Wien: WUV-Universitätsverlag 2000.

8 Vgl. Schmidt/Westerbarkey (wie Anm. 1), S. 249. 
Ein Bewusstsein für den konstruktivistischen Kontext („contexte constructiviste“) ihrer Wissenschaft haben die französischen Semiotiker durchaus, und zwar sowohl innerhalb als auch außerhalb der Disziplingrenzen der Kommunikationswissenschaft. ${ }^{9}$ So führt der französische Semiotiker Landowski aus, ein „approche constructiviste“, ein konstruktivistischer Ansatz, meine zunächst nur, dass Realität, verstanden als Sinn niemals präexistent sei. Sinn entstehe immer durch einen Setzungsakt, vor allem als ein Akt des Setzens von Werten, sei es im Falle diskursiver Mechanismen oder der metadiskursiven der Semiotik selbst. ${ }^{10}$ Ein ontologischer Seins- und Wertbegriff ist hier aufgegeben. ${ }^{11}$ Aufgabe der Semiotik sei es gerade, Konstruktionsprozesse sichtbar zu machen. Boutaud findet in diesen Ausführungen Landowskis die erkenntnistheoretische Bedeutsamkeit der Semiotik für eine Wissenschaft von der Kommunikation: Kommunikation ist weniger „échange“ (Tausch) als „co-production“.12

Innerhalb der französischen Kommunikationswissenschaft fällt darüber hinaus eine kritische Auseinandersetzung mit der postmodernen Beschreibung des „rasenden Stillstandes" (Virilio) und ähnlicher Diagnosen auf. Auch diesbezüglich gibt bereits ein einzelnes Buch Auskunft, das u. a. den neueren Stand der semiotischen Forschung in der französischen Kommunikationswissenschaft zusammenfasst, „Sémiotique et communication. Du signe au sens", das Buch von Jean-Jacques Boutaud, der an der Université de Bourgogne „Sciences de l'information et de la communication“ lehrt.13 Denn so heißt das Fach in Frankreich an nahezu allen Universitäten, an denen es gelehrt wird: Dass „information“ als Zeichenübertragungsprozess („transmission“) - auch von bits und bites - und „communication“ im Sinne des Zusammenkommens von Menschen und damit elementar von sinnstiftender („sens“) Humankommunikation mit all ihren Unabwägbarkeiten, offenbar doch etwas ganz Unterschiedliches sind, das lernt man schon in einschlägigen französischen Einführungslektüren, etwa von Daniel Bougnoux. ${ }^{14}$ Daher rührt auch eine sehr kritische Auseinandersetzung mit Shannon und Weavers Informationstheorie zuallererst, mit Transmissionsansätzen grundsätzlich, und in der Folge eine Hinwendung zur Sprache, zur Zeichengebung und ihrer spezifischen Bedeutung in interaktiven Kontexten. Sowie nicht zuletzt der Blick auf die Medien als uns umgebende und diese Umgebung verändernde Alltagskultur, die wiederum Anlass zu semiotischen Fragestellungen gibt, aber auch zu einer Kulturphilosophie über die Medien. In Frankreich hat man sich ungleich stärker und länger mit Marshall McLuhans „hot“ and „cold media“ als bei uns beschäftigt. ${ }^{15}$ Die deutsche „Medienwissenschaft" findet neuerdings ihr Pendant in der „Mediologie“, Daniel Bougnoux und Régis Debray sind ihre nam-

9 Zum „contexte constructiviste“ vgl. Boutaud (wie Anm. 6), S. 92.

10 Edgar Landowski: Le semioticien et son double. In: Lire Greimas. Limoges: Pulim, S. 229 255, 245.

11 Man fühlt sich an Luhmanns ebenso antimetaphysische Prämisse der Beliebigkeit von Sinn erinnert. Vgl. Niklas Luhmann: Soziale Systeme. Grundriss einer allgemeinen Theorie. Frankfurt a. M.: Suhrkamp 1987, S. 92 ff.

12 Vgl. Boutaud (wie Anm. 6), S. 92.

13 Vgl. Boutaud (wie Anm. 6).

14 Vgl. Daniel Bougnoux: Introduction aux Sciences de l'information et de la communication. Paris: Edition La Découverte 1998, S. 8 ff. Vgl. auch ders.: La Communication contre l'information. Paris: Hachette 1995.

15 Vgl. insbesondere Françis Balle: Pour comprendre les médias. Mc Luhan. Analyse critique. Paris: Hatier 1972. 
haften Vertreter, ${ }^{16}$ die allerdings von eher funktionalistisch orientierten Denkern wie Francis Balle, immerhin selbst ein guter Kenner (und Kritiker) McLuhans, ebenso heftig abgelehnt wird wie von Kommunikationssemiotikern wie Boutaud; mit unterschiedlichen Begründungen: Die Médiologie vernachlässige das menschliche Handeln, insbesondere auf politischer Ebene, so Balle; die Médiologie blende die Eigenwertigkeit von Symbolprozessen aus, indem sie diese kausal an bestimmte Transmissionsmöglichkeiten binde (etwa in der McLuhan nachgebildeten Einteilung Debrays oraler, grafologischer und vom Bildschirm bestimmter Kulturepochen), so Boutaud.17 Wieweit diese Mediologie nicht nur dem Namen, sondern auch den Inhalten nach einer deutschen „Medienwissenschaft" nahe kommt, ist - selbstredend - nicht untersucht.

Was wir nicht über die Franzosen wissen, wissen sie auch nicht über uns: Die deutsche Kommunikationswissenschaft wird in Frankreich nur sehr marginal wahrgenommen, ${ }^{18}$ die einzige Fachvertreterin, die relativ breit rezipiert wird, ist Elisabeth Noelle Neumann, ${ }^{19}$ Jürgen Habermas (selbstverständlich nicht als „deutscher Kommunikationswissenschafter“, sondern als Soziologe und Philosoph) ebenfalls, 20 beide sitzen im Beirat der kommunikationswissenschaftlichen Zeitschrift Hermès. ${ }^{21}$ Niklas Luhmann findet - auch nach einem Luhmann-Schwerpunkt der renommierten Fachzeitschrift Réseaux - kaum Resonanz.22 Warum? Meine These lautet, das systemische Denken ist „den

16 Vgl. Régis Débray: Cours de médiologie génerale. Paris: Gallimard 1991; ders.: Manifestations médiologiques. Paris: Gallimard 1994. Sowie die von Debray herausgegebene und von Bougnoux als Redakteur betreute Zeitschrift Les Cahiers de médiologie; insbesondere No. 6 / Deuxième Semestre 1998 die Sondernummer „Pourquoi des médiologues?“

17 Interview d. Verf. mit Françis Balle in Paris am 9.2.1999 sowie Boutaud (wie Anm. 6), S. 84.

18 Dies ergab auch das Interview Françis Balle im Februar 1999 in Paris. - Verwiesen sei allerdings auf eine durchaus reichhaltige Rezeption der frühen deutschen Soziologie, insbesondere der „Frankfurter Schule“; die Verf. selbst hat diesbezüglich im ersten Trimester 1999 eine Vorlesung von Rémy Rieffel an der Universität Paris II über Walter Benjamin und Max Horkheimer und die Relevanz ihrer Werke für kommunikationswissenschaftliche Fragestellungen gehört. Auch diese Dimension französisch-deutscher Wahrnehmung ist bislang unreflektiert.

19 Indes ist das Buch über die „Schweigespirale“ nicht in die französische Sprache übersetzt, französischen Forschern aber über die englischsprachige Fassung zugänglich. Noelle-Neumann selbst stellte gemeinsam mit Wolfgang Donsbach in einem französischen Sammelband die deutsche Kommunikationswissenschaft vor; mit deutlichem Schwerpunkt auf der Theorie der Schweigespirale. Vgl. Elisabeth Noelle-Neumann/Wolfgang Donsbach: Allemagne Fédérale. In: Les Cahiers de la Communication, Vol. 2 (1982), No. 4 - 5 (Numéro: Communications et médias. Les orientations de la recherche en Europe), S. $301-325$.

20 Vgl. vor allem Jean Marc Ferry: Habermas. L' éthique de la communication. Paris: PUF 1987.

21 Vgl. Hermès. Cognition, Communication. Politique. Quadrimestriel. Edition du Centre National de la Recherche Scientifique (C.N.R. S.). Paris. Directeur: Dominique Wolton: Conseil Scientifique [1999]: Paul Amselek, Marc Auge, Jay G. Blumer, Bario Borillo, Roland Cayrol, Jean Pierre Desceles, Jean Pierre Dupuy, Marc Ferro, Max Fontet, Jürgen Habermas, Elihu Katz, Jean Leca, Jean-François Lyotard, Lous Marin, Edgar Morin, Elisabeth Noelle-Neumann. Die Zeitschrift erscheint seit 1988.

22 Vgl. Réseaux. Communication. Technologie. Société. Revue bimestrielle. Edité par le Groupe de Recherche (G.D.R), Communication du Centre National d'Etude des Telecommunication (C.N.E.T.) et du Centre National de la Recherche Scientifique (C.N.R.S.). Rédacteurs en chef: Paul Béaud/Patrice Flichy. Die Zeitschrift erscheint seit 1983. - Auf die stark zentralistische Organisation von Forschung, die sich in der Anbindung beider Zeitschriften, Hermès und Réseaux, an das C.N.R.S zeigt, kann hier nicht eingegangen werden. Vgl. Niklas Luhmann: Communication et Action. In: Réseaux, No. 50, Nov./Dec. 1991, S. 131 - 156. 
Franzosen“ aus erkenntnistheoretischen Überlegungen heraus zu markroperspektivisch, zu wenig subjektorientiert. Als sinngebende Instanz wird vor allem das Subjekt angesehen. Dies gilt insbesondere für die Richtungen, die die amerikanischen Traditionen und Perspektiven weit kritischer rezipieren als wir, wiederum insbesondere die semiotische Richtung der französischen Kommunikationswissenschaft, exemplarisch sei hier noch einmal Boutaud benannt, aber gleiches gilt etwa auch für Eliséo Véron, einen Wissenschaftler argentinischer Herkunft, der jahrelang in Paris gelehrt hat und in Frankreich stark wahrgenommen wird.

Die französische Kommunikationswissenschaft kennt - anders als Ulrich Saxer dies nahe legt ${ }^{23}$ - eine breite Auseinandersetzung mit der angelsächsischen Tradition. ${ }^{24}$ Ethnozentrisch motiviert ist allenfalls die kritische Auseinandersetzung mit der angelsächsischen Tradition selbst - allein die Analyse dieser kritischen Auseinandersetzung würde sich als Untersuchungsgegenstand sicher lohnen und nationale Verschiedenartigkeiten und Gemeinsamkeiten unseres Faches stärker erhellen.

Insgesamt gilt für Frankreich - glaubt man Françis Balle, der als Professor für Kommunikationswissenschaft an der Universität Panthéon-Assas in Paris, als Gastdozent an der Sorbonne ebenso wie als Leiter des in Paris ansässigen „Institut de Recherches et d'Etudes sur la Communication" die Szenerie immerhin von innen kennt: Das Fach begann sich Anfang der 60er Jahre zu konstituieren und war zunächst sowohl von marxistischen Richtungen als auch vom amerikanisch-funktionalistischen Paradigma stark geprägt. Es bildeten sich kaum Schulen, es waren Einzelforscher, die diese Richtungen vertraten. Die marxistische Orientierung - einhergehend mit poststrukturalistischen Theorieansätzen - sei mit dem politischen Zusammenbruch des Marxismus im Jahre 1989 zunehmend irrelevant geworden. Dies lasse auf neue Ansätze und eine Verringerung der Grabenkämpfe zwischen empirisch-funktionalistischen und kritisch-materialistischen Denkern hoffen.25 Das Buch von Boutaud, die Entmystifizierung des semiotischen Ansatzes von einer neomarxistischen Normbasis, 26 bestätigt diese Hoffnung.

Die Erforschung der eigenen Tradition ist in der französischen Kommunikationswissenschaft sicherlich geringer ausgeprägt als in Deutschland: So liegt bislang keine systematische fachhistorische Aufarbeitung der französischen Kommunikationswissenschaft vor, und bis auf wenige Passagen in einzelnen Aufsätzen und einige (verschriftlichte) Interviews mit französischen „Gründervätern“ dieses Faches kaum Sekundärquellen zur Institutionalisierung. ${ }^{27}$ Bis heute tendiert das Selbstverständnis der „Sciences de l'information et de la communication “ eher zu dem eines interdisziplinären Forschungsfeldes, als dem einer wissenschaftlichen Fachgemeinschaft.

23 Vgl. Saxer (wie Anm. 1), S. 86.

24 Vgl. insbesondere Judith Lazard: La Science de la communication. Paris: PUF 1992; Armand Mattelart/Michèle Mattelart: Historie des théories de la communication. Paris: Edition La Découverte 1995.

25 Interview d. Verf. mit Françis Balle am 9.2.1999 in Paris.

26 Die neo-marxistische Sicht entwickelte sich vor allem am „Centre d'Etudes de Communication de Masse“ (C.E.C.M.A.S). Hier arbeiteten Georges Friedman, Roland Barthes (der als Begründer der zeitgenössischen französischen Semiotik gilt) und Christian Metz (der als Begründer der Filmsemiotik gilt); sie edierten die Zeitschrift Communications. Mit dem Tod Georges Friedmans verlor die Forschergruppe Ende der 70er Jahre ihren Zusammenhalt.

27 Vgl. etwa: Klaus Bruhn-Jensen/Karl Erik Rosengreen: Cinq traditions à la recherche du public. In: Hermès 1992, No. 11 - 12 (A la recherche du public), S. 281-300; Olivier Burgelin: Structural analysis and Mass Communications. Tendency of French Research on Mass Com- 
Eine der zentralen Lehr- und Forschungsstätten ist das Institut Français de Presse in Paris. 1951 an der Juristischen Fakultät der Université Paris II begründet, beherbergt das Institut heute mit über 8000 Werken, nahezu vollständigen Beständen einschlägiger Fachzeitschriften und einer breiten Pressedokumentation die wohl umfassendste Sammlung zur französischen Kommunikationswissenschaft in Frankreich. Längst ist es nicht mehr rechts-, sondern sozialwissenschaftlich verortet. Direktor des Instituts war bis 1999 Rémy Rieffel,28 spezialisiert auf Journalismusforschung und Kommunikationstheorien. Rieffel ist in Deutschland durch seine enge Zusammenarbeit mit Ursula E. Koch, meines Wissens eine der wenigen deutsch-französischen Kooperation in beiden Ländern auf kommunikationswissenschaftlichen Gebiet, bekannt geworden. Gemeinsame Tagungen begleiten diese Zusammenarbeit. Über das Gebiet der (vergleichenden) Journalismusforschung hinaus, ${ }^{29}$ wird in Kürze in Paris der Tagungsband des Kolloquiums „Neue Ansätze der Publikumsforschung in Deutschland und Frankreich“, das Ende 1998 im kommunikationswissenschaftlichen Institut der Universität München stattfand, in Paris erscheinen. Die Kolloquiumsbände lösen das Sprachproblem durch Bilingualität - alle Texte sind jeweils sowohl in französischer als auch in deutscher Sprache abgedruckt.

\section{Der „contexte constructiviste“ oder: Kritik am Postmodernismus}

Die Auseinandersetzung der französischen Kommunikationswissenschaft mit der französischen postmodernistischen Soziologie und Philosophie und ihrer Betrachtung von „Kommunikation“ steht in den Anfängen. Statt anderer soll hier die Position von Jean Jacques Boutaud skizziert werden. ${ }^{30}$

Es gibt Realität, insoweit sie das Sprachhandeln selbst ist. Die Postmodernisten, die nahezu ausschließlich subjektzentriert und zudem fixiert auf die geistige „Wüste“, die der „Information overload“ vermeintlich hinterlasse, blickten, vergäßen, so Boutaud, auf den Akt der Kommunikation zu schauen. Statt auf die Kommunikation zwischen und von Menschen (auch soweit diese über Medien geschieht) zu referieren, versuchten sie die Synchronität und Überkomplexität von Information begrifflich zu fixieren. Das eigentliche Ziel kommunikationswissenschaftlicher Analysen - die Abläufe und Bedeutungsebenen jeder Form von Humankommunikation, ob vermittelt oder unvermittelt, träfen sie nicht. Boutaud schlägt stattdessen vor, Information und Kommunikation ergänzend zu betrachten, eine Perspektive, die auch Daniel Bougnoux vertritt. Information ist dabei höchstens Mitteilung, aber noch keine Kommunikation, die erst durch den Akt der Interpretation (Reflexion des Subjekts auf sich selbst und seine Beziehung zu anderen) entstehe. Ein Zeichen erhält Bedeutung erst durch die Symbolisierung, die es

munications. In: Studies of Broadcasting, No. 6, S. 143 - 168; statt anderer auch das Interview von Jean Devèze mit Robert Escarpit, Gründungsmitglied der „Société française de l'information et de la communication“ (SFSIC), Les fondateurs de la SFSIC: Robert Escarpit. Edité par SFIC. Ohne Jahr [1993].

28 Die derzeitige Direktorin ist Nadine Toussaint-Desmoulins. Vgl. die Institutshomepage (auch in englischer Sprache): http://www.u-paris2.fr/ifp

29 Vgl. etwa den aus dieser Zusammenarbeit hervorgegangenen Sammelband von Ursula Koch/Detlef Schröter/Pierre Albert/Rémy Rieffel (Eds.): La Radio en France et en Allemagne. Un dialogue entre journalistes et chercheurs. Munich: Reinhard Fischer 1996.

30 Vgl. zu den folgenden Ausführungen Boutaud (wie Anm. 6) sowie ergänzend Bougnoux (wie Anm. 14). 
aufgrund sozialer Kontexte und interaktiver Zusammenhänge erfährt. Dies ist gemeint, wenn Boutaud und Landowski (siehe oben) vom "contexte constructiviste“ der Kommunikation sprechen; Kommunikationsprozesse sind kreativ und re-kreativ, sowohl auf Seiten des Kommunikators wie des Rezipienten.

Hier tut sich auch der Unterschied zur Linguistik auf: Sie ist (in erster Linie) an Zeichensystemen interessiert und nicht an Sprachpragmatik. Folglich macht sich der Zusammenhang Semiotik - Kommunikationswissenschaft erst mit Charles Sanders Peirce und seiner Einführung des Sprachhandelns und des umgebungsbezogenen Subjektes auf. So weit Jean-Jacques Boutaud.

Boutaud macht folgendes Szenario auf: Es gebe eine soziologische und psychologische Realität, an der der Semiotiker ${ }^{31}$ aber nicht in erster Linie interessiert sei. Er sei vielmehr an der Repräsentation dieser Realität durch Zeichen und ibrer Verwendung im Rahmen des Kommunikationsprozesses interessiert. Verwendung meint sowohl die des Kommunikators wie die des Rezipienten. Empirischer Bezugspunkt der Semiotik sei die in Zeichen und Symbolen repräsentierte Realität und der - durch den Semiologen - rekonstruierte Kommunikator wie Rezipient. Letztere bleiben empirisch rückbezüglich, rekonstruierbar, über ihre aktive Verwendung von Zeichensystemen, von Formen. Diese „Formen“ sind kontextuell von Kulturräumen, Kommunikationssituationen, abhängig, sodass sich aus ihnen wiederum Generalisierungen über kommunikatives Verhalten, im Sinne von Bedeutungszuweisungen innerhalb einer bestimmten empirisch vorhandenen menschlichen Gemeinschaft, ableiten lassen.

Nicht anders als in der Moderne und in der Vormoderne verfügt auch der „postmoderne“ Mensch über solche realitätsrückbezüglichen Selektions- und Konstruktionsstrategien. Es hat sich nichts geändert, kein „Ende der Geschichte“ ist in Sicht, und auch das Ziel der Kommunikationswissenschaft: die Mechanismen der Sinnkonstruktion aufzudecken, ist noch nicht erreicht.

„Konstruktion“ meint in dieser Lesart vor allem die Konstruktion von Sinn durch Zeichensysteme, nicht eine bestimmte erkenntnistheoretische Bezugnahme auf die Differenz Realität versus Nichtrealität. Die Realität als bestehender Bezugsfundus aller wird nicht angezweifelt; und doch tun sich hier Denkmotive auf, wie sie sich auch im (radikalen) Konstruktivismus finden: Realität wird über Repräsentation erfabrbar. Daher kann und muss der Forscher diese Repräsentation und ihre Ausprägungen untersuchen und findet hier den elementarsten Ansatzpunkt seiner Untersuchung. Repräsentation ist nicht beliebig. Sinngebung ist an Repräsentationen rückgebunden. Sinngebung wird dabei von Boutaud weder metaphysisch noch sensualistisch, behavioristisch oder instinktgebunden beschrieben. All das aber bleibt - als anthropologisches Umfeld der Repräsentation - im Horizont der semiotischen Analyse, aber liegt nicht in deren Zentrum: Im Mittelpunkt einer kommunikationswissenschaftlichen Analyse auf semiotischer Basis steht die Repräsentation von Sensationen, Trieben, Ideen, Ideologien. Denn auf dieser Repräsentation beruht Kommunikation; Kommunikation ist aber als Interaktion nicht mit Repräsentation identisch. Kommunikation knüpft an Repräsentation an. Das etwa wusste auch der symbolische Interaktionist George Herbert Mead oder Er-

31 In Frankreich werden die Begriffe „sémiotique“ / „sémioticien“ (Semiotik/Semiotiker) und „sémiologie“ / „sémiologue“ (Semiologie, Semiologe) ebenso wie in Deutschland parallel verwandt: Die Semiologie meint dabei eher die klassische, linguistisch und sprachphilosophisch orientierte Lehre von den Zeichen; die Semiotik eher pragmatisch ausgerichtete Ausdruckslehren. Boutauds Buch (vgl. Anm. 6) heißt auch daher "Sémiotique et communication“. 
ving Goffmann in seiner Rollentheorie; Letzterem fühlt Boutaud sich besonders verbunden.

Boutauds Ansatz, die Semiotik an sozialpsychologische und soziologische Theorien zu binden, sie - in ihrer Bedeutung für die Erforschung von Kommunikation - sozialwissenschaftlich wenden zu wollen (Frage nach der Bedeutungsgebung innerhalb sozialer Kontexte) steht nicht allein; andere Autoren, die sich um das gleiche Problem bemühen sind etwa Eliséo Véron, Alex Muchielli, Erik Fouquier, Robert Ghiglione und Frédéric Lambert. 32

Diese die Grenzen von Semiologie und Soziologie überschreitende Richtung ist meines Erachtens eine viel versprechende Richtung der französischen Kommunikationswissenschaft, die auch international von hohem Interesse sein könnte - wenn sie denn bekannter wäre.

In Frankreich selbst wird den „Semiologen“ unter den Kommunikationswissenschaftlern mitunter der Vorwurf gemacht, sie seien „message“-fixiert. Die Mitteilung stehe im Zentrum der Betrachtung, nicht die Produktionssituation, nicht die Rezeptionssituation, nicht der Produzent, nicht der Rezipient. Boutaud, Bougnoux, Véron und andere gehen indes seit Mitte der 80er Jahre davon aus (und lehnen sich darin vor allem an die Sprachpragmatik John L. Austins an), dass dies nicht zwangsläufig so sein muss, sondern sich Semiotik - vermittelt über das repräsentierende Zeichen - auch auf Interaktionen beziehen lässt: Sowohl der Produzent als auch der Rezipient weisen einer Mitteilung selbsttätig Sinn zu, vor einer bestimmten kulturellen Folie, die für Produzent und Rezipient übereinstimmen kann, dies aber nicht zwangsläufig muss (spätestens dann kommt es zu den von Watzlawick beschworenen Missverständnissen). Dieser Ansatz bedeutet auch eine methodologische Erweiterung. Die semiologische Analyse bezieht sich dann nicht mehr nur auf die „message“, sondern auch auf das Bedeutungsverständnis ihrer Leser/Hörer/Zuschauer. Eliséo Véron, bis Ende der 90er Jahre Professor für Kommunikationswissenschaft in Paris wie Direktor eines kommerziellen Instituts im Bereich der Marktforschung, schlägt vor, und hat dies auch selbst schon unternommen, klassische semiologische Analysen von Zeitungsinhalten künftig stärker mit Leserbefragungen zu kombinieren, wobei der Fragebogen anhand der in der semiologischen Analyse gewonnen Erkenntnisse etwa über Sinnkontexte zu formulieren ist. ${ }^{33}$

Das Ziel geht über soziologische Analysen zu Leserstrukturen und -bewegungen hinaus und zielt auf das konstruktive, ergo das kommunikative Potenzial des Lesers (oder Hörers oder Betrachters).

32 Vgl. Eric Fouquier/Eliséo Véron: Les spectacles scientifiques télévisés. Figures de la production et de la réception. Paris: La Documentation Française 1986; Robert Ghiglione: L'homme communiquant. Paris: Armand Collin 1986; Jacques Leenhardt: Théorie de la communication et théorie de la reception. In: Réseaux, No. 68 Nov./Dec. 94, pp. 41 - 48; Alex Muchielli: Sciences de l'information et de la communication. Paris: Hachette 1995. Auf weitere Literaturangaben soll verzichtet werden; vgl. die systematisch gegliederten Übersichten der gut sortierten kommerziellen kommunikationswissenschaftlichen Buchhandlung „Teckhné“ in Paris: Médias. Le Livre des Livres, édité par Teckhné 1997ff. (dieser Bücherkatalog umfasst 800 Werke).

33 Vgl. dazu Nathalie Robert: Sémio. Tout est Discours. In: Mega-Bilan: La Recherche Marketing et la Sémiologie. In: Médias, No. 77, Mai 1984, S. 67 - 70 sowie Eliséo Véron: Les médias en réception. Les enjeux de la complexité. In: Médiaspouvoirs, No. 21 Jan/Fev. 1991, S. $165-173$. 


\section{Ausblick}

Dieses Schlaglicht auf die französische Kommunikationswissenschaft speist sich aus kursorischer Lektüre und bedarf der Fundierung. So viel aber steht fest: Es geht nicht an, die Kommunikationswissenschaft in unserem Nachbarland nicht wahrzunehmen (ebenso wenig sie auf eine Perspektive „Postmoderne“ zu reduzieren). Es gilt sie zu studieren. Denn die Grundfrage, die sie stellt, ist gleichsam international bedeutsam: Wie entsteht sozialer Sinn in Gesellschaften über Kommunikation? Eine sehr breite Richtung innerhalb der französischen Kommunikationswissenschaft stellt diese Frage für das Feld der „communication politique“, der politischen Kommunikation. ${ }^{34}$

Komparatistische Fragestellungen an die Kommunikationswissenschaft in Frankreich und in Deutschland bzw. im deutschsprachigen Raum zu stellen, ist möglich. Und hier tut sich auch gleich eine weitere Perspektive auf: Denn Frankreich blickt in seiner kommunikationswissenschaftlichen Literatur, abgesehen von den eher kritisch reflektierten USA, vor allem in die Frankofonie, besonders nach Belgien und Kanada, aber auch in die Romanie, ${ }^{35}$ nach Italien, nach Spanien. Wiederum weiße Felder in unserer Wahrnehmung oder: der blinde Fleck des Beobachters, der sich selbst nur im Spiegel des eigenen wissenschaftlichen und kulturellen Horizonts wahrnimmt und eben diesen Horizont zum Bewertungsmaßstab macht.

Selbstverständlich hat eine Wissenschaft keinen „Nationalcharakter“, aber, und dies scheint sich im Falle der französischen Kommunikationswissenschaft - gemessen am „Maßstab“ der Deutschen (oder umgekehrt ...) - zu bestätigen, doch spezifische Charakteristika. Diese ergeben sich (auch) aus verschiedenen wissenschaftssoziologischen und -historischen Bedingungen 36 in den Ländern. Länderübergreifende Theorievergleiche sind komplex und aus heuristischen Gründen sicherlich zugleich vereinfachend. Im Sinn haben sollten sie die Erkenntnis der eigenen Rahmenbedingungen der Wissenschaftsentwicklung, die sich oft erst im Vergleich erschließt, ${ }^{37}$ sowie der Möglichkeit neue Fragestellungen zu entwickeln, oder ganz schlicht: voneinander zu lernen.

34 Vgl. übergreifend Jacques Gerstlé: La Communication politique. Paris: PUF 1992 (Edition Que-sais-je?).

35 Hier sei nur darauf hingewiesen, dass nicht nur die rumänische Sprache, sondern auch die rumänische Kommunikationswissenschaft dem Französischen eng verbunden sind. Vgl. etwa die von Mihai Coman herausgegebene und von Daniela Roventa-Frumusani, Professorin an der Fakultät für Journalistik und Kommunikationswissenschaft in Bukarest, redaktionell betreute Zeitschrift Le Résean Global. Communication et Société en Europe de l'Est. Die Zeitschrift führt zugleich den englischen Titel The Global Network. Communication and Society in Eastern Europe.

36 Hier ist sicherlich die (Selbst)instrumentalisierung der Zeitungswissenschaft des „Dritten Reiches“ für die Entwicklung der bundesrepublikanischen Kommunikationswissenschaft und ihre Anpassung an amerikanische Standards nicht außer Acht zu lassen.

37 Vgl. etwa den komparatistischen Ansatz, mit dem Haas die Entwicklung der Zeitungswissenschaft in Deutschland und der Kommunikationsforschung in den USA analysiert und bewertet. Hannes Haas: Zeitungswissenschaft und Communications Research 1918 - 1945. Ein methodologischer, theoretischer und paradigmatischer Vergleich. In: Oliver Rathkolb/Wolfgang Duchkowitsch/Fritz Hausjell (Hrsg.): Die veruntreute Wahrheit. Hitlers Propagandisten in Österreichs Medien. Salzburg: Müller 1988, S. 252 - 272, 497 - 482. 